\title{
Un nouveau foyer pour enfants abandonnés
}

\section{Gottfried Huss}

Dr med., MPH, Conseil de I'association suisse

Pourquoi un village d'enfants est-il nécessaire au Maroc? Celui qui visite ce pays découvre que le Maroc est un beau pays et que sa société est pleine de contradictions. Dans ce royaume, les traditions et la religion sont vivaces, par contre il n'y a malheureusement pas de place pour les mères célibataires et leur progéniture. Au Maroc, rien n'est plus honteux (Hchouma) pour une femme que de donner le jour à un enfant illégitime. Les mères célibataires sont discriminées tant

Au pied de l'Atlas, non loin de Marrakech, sur un terrain de dix hectares est né un village.

par la société que par la loi. La pression de l'environnement est telle que les mères, souvent très jeunes, font naître leur bébé dans le secret et s'en débarrassent aussitôt. Le nombre d'enfants abandonnés est en augmentation: on estime qu'ils sont entre 8000 et 10000 , mais il n'existe aucune statistique officielle. Le sujet est tabou et il est exclu d'en parler à haute voix. Cette atteinte larvée aux droits des enfants est aussi éclatante qu'inacceptable.

\section{Un Suisse qui vient en aide}

Le profil de Hansjörg Huber, un Zurichois, ancien professionnel des assurances, se caractérise par trois éléments: avoir des moyens, un grand cœur pour l'enfance malheureuse et être plein d'énergie. «Il s'agit de chérir ces enfants», dit-il. Il a commencé en 2008 de construire de ses propres moyens tout un village d'enfants. Au pied de l'Atlas, non loin de Marrakech, sur un terrain de dix hectares est né un village en mesure d'offrir à une centaine d'enfants un foyer, un environnement et une école leur assurant un avenir. Ce village consiste en 13 maisons d'habitation, une école, une infirmerie, une mosquée, des maisons d'hôtes, une ferme-modèle, une galerie d'art et deux maisons médicalisées. L'une de ces dernières est destinée à la couverture des besoins médicaux de tout le village d'enfant

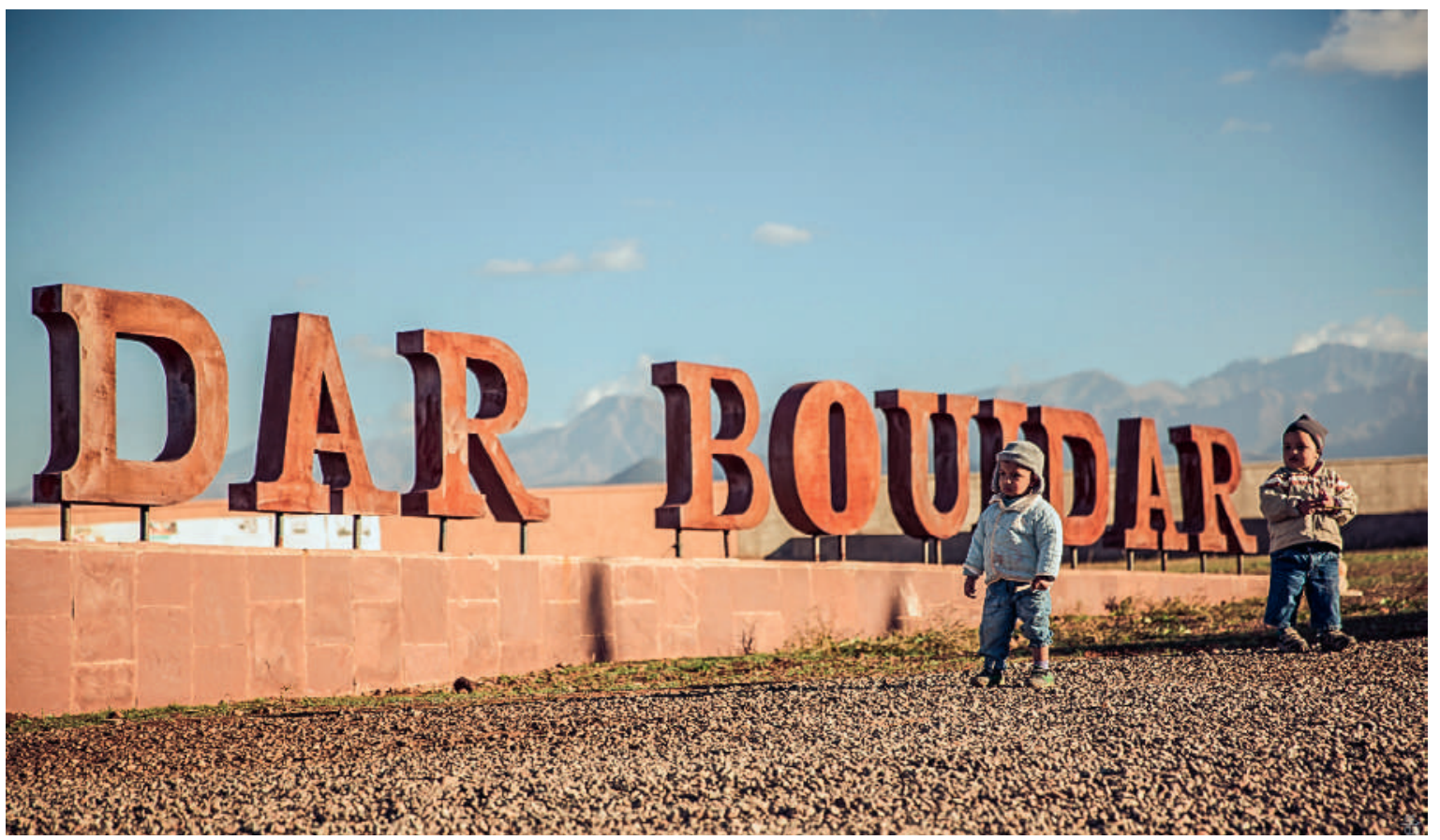

Figure 1: Entrée Dar Bouidar. 


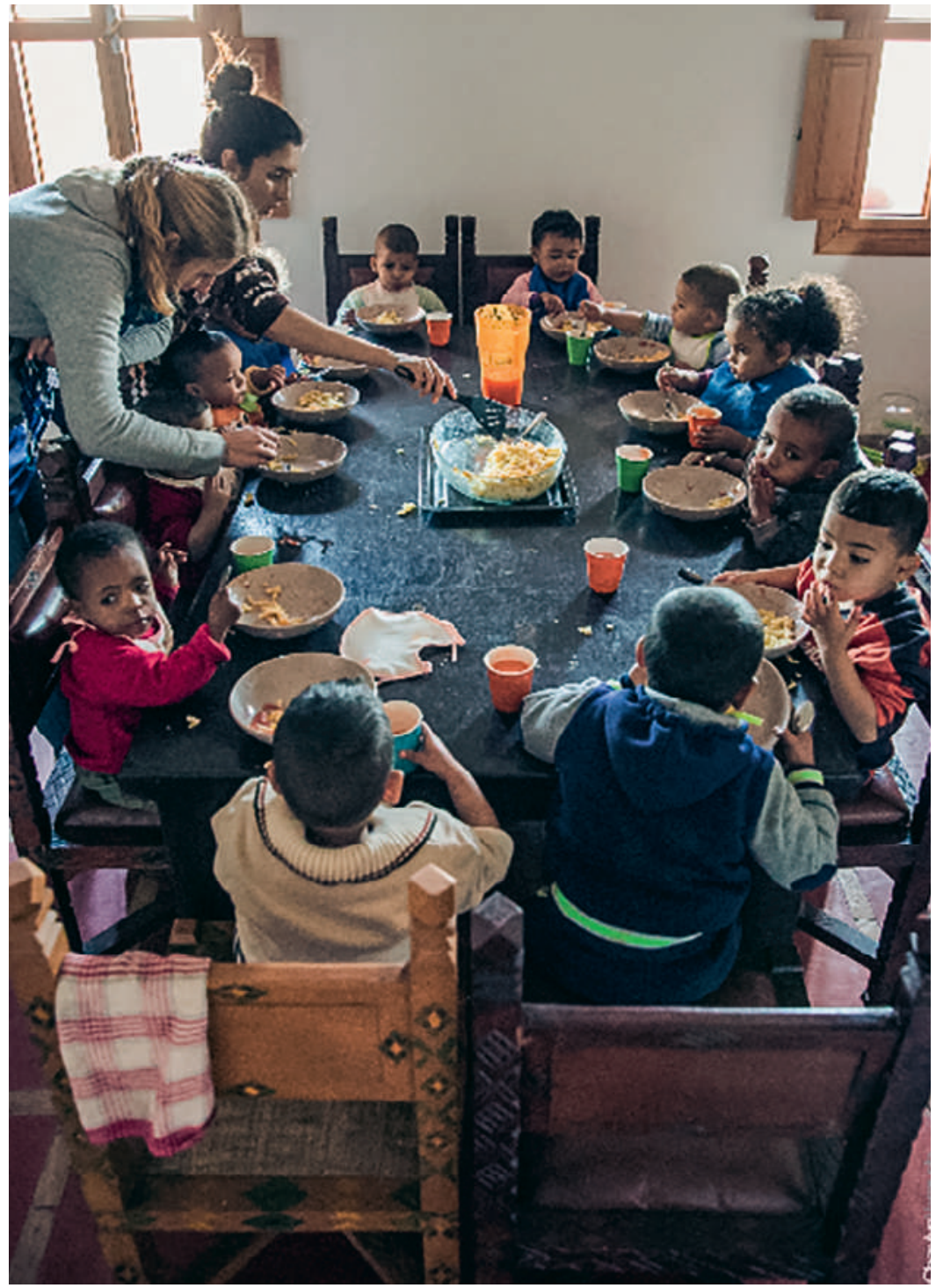

Figure 2: Prendre le déjeuner ensemble.

ainsi que de ceux du voisinage. L'autre sert aux consultations obstétriques, les femmes de la région n'ayant d'habitude pas d'accès aux contrôles de grossesse. Monsieur Huber est parvenu à obtenir du ministère de la santé une autorisation permettant l'engagement de

Il est garanti que tout don est consacré à $100 \%$ à la construction et à l'entretien du village d'enfants.

médecins étrangers à des fins humanitaires pour des durées n'excédant pas 30 jours. Après vérification de leurs qualifications professionnelles, ces médecins peuvent être envoyés dans les régions les plus reculées de l'Atlas où les populations locales accueillent avec reconnaissance l'assistance médicale faisant défaut. Une expérience unique! Le gîte et le couvert de ces médecins sont assurés.

\section{Les quatre piliers de base du village d'enfants et sa pérennité}

L'esprit de ce village d'enfants, spécialement orienté sur la famille, repose sur les principes de la Convention des droits de l'enfance de l'ONU. Quatre principes ont été définis sur cette base:

- Chaque enfant doit être relié à une personne responsable et digne de confiance, une «mère».

- Chaque enfant doit grandir de façon naturelle avec des «frères et sœurs".

- Chaque enfant doit pouvoir habiter une maison qu'il doit pouvoir considérer comme son foyer.

- Chaque enfant doit pouvoir vivre dans une atmosphère champêtre, propice à son développement.

Afin d'assurer la pérennité du village et de réaliser son intégration à la société marocaine, chaque étape a été conçue d'entente avec les autorités, et des structures durables ont été créées. Architectes, avocats, entrepreneurs, jardiniers, gardiens, mères, chauffeurs, enseignantes, infirmières: tous et toutes sont Marocains, berbères pour la plupart. Lâge de la scolarité atteint, les enfants sont envoyés à l'école de Tahanaoute, la localité voisine. Dans le premier des dix villages planifiés vivent actuellement 29 enfants. D'ici fin 2016, ils devraient être 100.

\section{De quelle sorte d'aide le village a-t-il besoin?}

«Fais le bien et parles-en» (dit le fondateur Hansjörg Huber). Si vous vous trouvez au Maroc, vous êtes le bienvenu au village. Contact: huberhansjoerg[at]gmail. com

Les associations d'entraide de Suisse, France et Allemagne qui travaillent à ce projet sont contrôlées par PricewaterhouseCoopers ( $\mathrm{PwC}$ ). Tous les administrateurs et les différentes aides travaillent à titre bénévole. Il est garanti que tout don est consacré à 100\% à la construction et à l'entretien du village d'enfants. Des pédiatres et des femmes médecins spécialisées en obstétrique peuvent s'annoncer pour un engagement minimum de trois semaines à l'adresse: info[at]kinder dorf-marrakech.ch

Crédits photo

(c) Cherylyn Vanzuela

D'autres informations peuvent être obtenues sur les sites www.kinderdorf-marrakech.ch

www.lesenfantsdarbouidar.com 